\title{
Simulation of helium and residue production in the Megapie target
}

\author{
S. Lemaire ${ }^{1, a}$, J.-C. David ${ }^{1}$, and S. Leray ${ }^{1}$ \\ DAPNIA/SPhN, CEA Saclay, 91191 Gif-sur-Yvette, France
}

\begin{abstract}
This work is aimed at characterizing the Megapie target in terms of main contributors to the activity, modification of the chemical composition as well as residue and volatile gas production. Calculations are performed with the nuclear transport code MNCPX2.5.0 coupled to the evolution code CINDER'90. In this work, we provide results for the lead-bismuth and its container. A sensitivity analysis with regards to the different models available in MCNPX2.5.0 as well as the effect of impurities is discussed.
\end{abstract}

\section{Introduction}

Spallation neutron sources have various applications, among which Accelerator Driven systems (ADS) considered as a possible option for the incineration of minor actinides. These systems couple a high intensity proton accelerator to a sub critical reactor through a spallation target. The subsequent multiplication of neutrons in the sub-critical nuclear reactor will then start the transmutation reactions. The advantage of such devices is that one can use nuclear fuels with high level of minor actinides, thus allowing high efficiency transmutation, which is not possible in current reactors because of safety concerns (delayed neutrons, temperature...).

Megapie (Megawatt Pilot Target Experiment) is a joint initiative by six European research institutes and JAERI (Japan), to design, build and demonstrate the feasibility of a liquid lead bismuth target for spallation facilities at a beam power level of $1 \mathrm{MW}$ at PSI (Switzerland). The target has been irradiated successfully during 4 months. The analysis of the results collected during the experiment is currently under progress. The comparison of these results with simulations will bring interesting lessons for future designs of spallation targets in terms of performances, resistance to damages, radioprotection of the target and evolution with time of the target characteristics.

To simulate such spallation target, generally, nuclear transport code packages are used and coupled to evolution codes. Transport codes usually consist of the coupling of a high energy part relying on spallation models and a low energy part utilizing nuclear data tables. The HINDAS program [1] contributed in providing simulation tools for ADS as reliable as possible or, at least, with a known uncertainty. In particular, improved models were delivered, namely INCL4 [2] for the intranuclear cascade and ABLA [3] for the evaporationfission, and implemented into the high energy transport code LAHET3 [4] and MCNPX [5].

In this work, we have performed calculations with the transport code MCNPX2.5.0 using the detailed geometry of the Megapie target [6], in order to estimate the main

\footnotetext{
${ }^{a}$ Presenting author, e-mail: sebastien.lemaire@gmail.com
}

contributors to activity and determine the respective contributions of spallation reactions and activation by low energy neutrons in the lead-bismuth and in the container. We will also present the modification of the chemical composition of the target. Then, the activity and the amount of radioactive volatile elements that can be released in case of a containment failure will be assessed. Calculations with different spallation models were done to assess their impact.

\section{Methodology}

Calculations were performed assuming an irradiation time (close to the actual one) of 120 days by $575 \mathrm{MeV}$ protons at $1.4 \mathrm{~mA}$. Impurities in $\mathrm{Pb}-\mathrm{Bi}$ as well as in the container were taken into account in the calculations and their effect on results assessed. Spallation residues produced through the primary interactions and secondary particles (down to $20 \mathrm{MeV}$ for neutrons and down to stopping for protons) are extracted using HTAPE3X from MCNPX2.5.0 and given to the evolution code CINDER'90 for subsequent decay. Different models have been considered to calculate the elementary cross-sections. In particular, the spallation models Bertini [7]-Dresner [8], INCL4-ABLA, CEM2k [9], ISABEL [10]-ABLA have been used. Residues produced by activation by the neutron flux below $20 \mathrm{MeV}$ are obtained by taking the neutron flux from MCNPX into CINDER'90. It has already been shown in the past [11] that the secondary activation of the spallation products being negligible, it was reasonable to assume a constant target composition.

\section{Results}

\subsection{Lead-bismuth (LBE)}

A large number of isotopes are contributing to the total activity in the $\mathrm{Pb}-\mathrm{Bi}$. It is confirmed by the contribution of two main elements, ${ }^{203} \mathrm{~Pb}$ and ${ }^{206} \mathrm{Bi}$, after one day of irradiation which accounts for less than $8 \%$ of the total activity of the LBE as can be seen in figure 1 . The activity decreases slowly from around $2.9 \times 10^{5}$ Curies just after irradiation. For long decay 


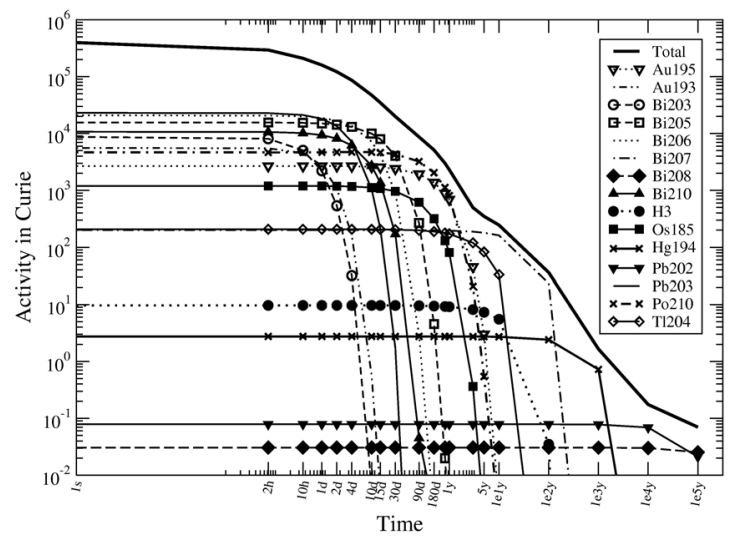

Fig. 1. Main contributors to the activity (in Curies) of the Megapie target as a function of cooling time after 120 days of irradiation with $575 \mathrm{MeV}$ protons at $1.4 \mathrm{~mA}$ using INCL4-ABLA models.

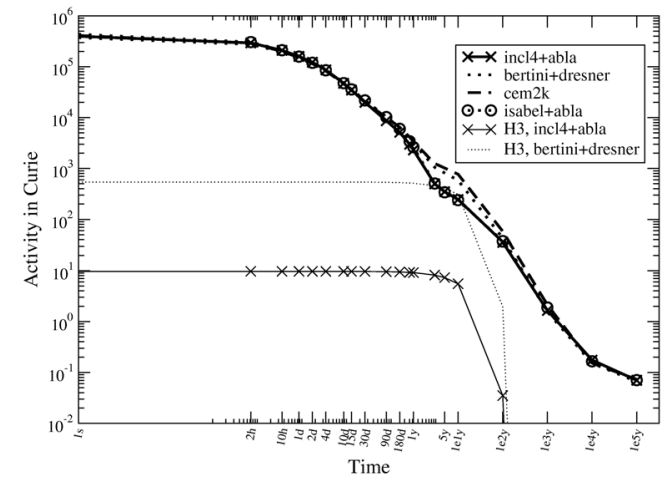

Fig. 2. Total activity (in Curies) of the LBE as a function of cooling time after 120 days of irradiation with $575 \mathrm{MeV}$ protons at $1.4 \mathrm{~mA}$ using different models.

times, activity is due to fewer nuclear elements such as ${ }^{194} \mathrm{Hg}$ (lifetime of 520 years), ${ }^{202} \mathrm{~Pb}$ (lifetime of $5.25 \times 10^{4}$ years) and ${ }^{208} \mathrm{Bi}$ (lifetime of $3.68 \times 10^{5}$ years) which represent $50 \%$ of the total activity. It is also interesting to mention that the main contributors to the activity of the liquid lead-bismuth target find their origin in heavy residues close to the target elements.

As shown in figure 2, the different spallation models used to compute the total activity in the LBE, give very similar results except in the region lying between 5 and 10 years of cooling time. It is identified that the differences obtained are due to the fact that the ABLA de-excitation model does not allow for evaporation of tritium contrary to Dresner or CEM2k. Contributions to the activity due to tritium in Bertini-Dresner and in INCL4-ABLA (coming only from Fermi breakup of light impurities) are also shown in figure 2. The chemical composition of the liquid metal target at the beginning of irradiation taking into account all the impurities in the $\mathrm{Pb}$ $\mathrm{Bi}$ and after 120 days is shown in figure 3 using different models. This is important since additional impurities produced by spallation reactions can lead to corrosion problems on the container of the target or with the window. Large differences are found between INCL4-ABLA and Bertini-Dresner models

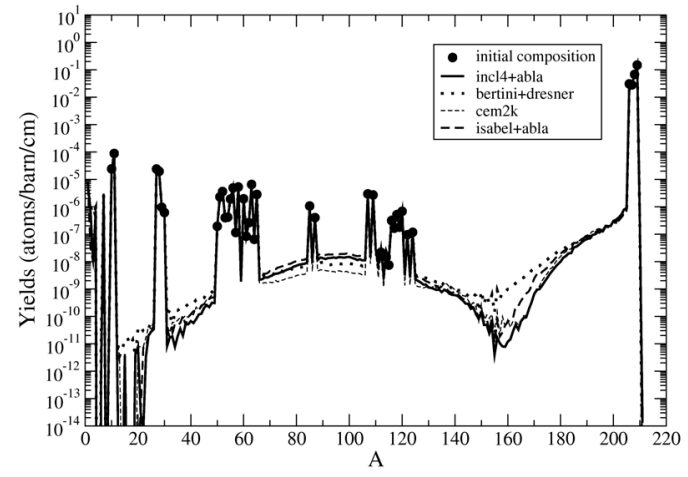

Fig. 3. Concentration of elements in the liquid $\mathrm{Pb}-\mathrm{Bi}$ target after 120 days of irradiation by $575 \mathrm{MeV}$ protons at $1.4 \mathrm{~mA}$. The initial composition of the target is represented as full black dots. Results obtained are shown using different spallation models: INCL4-ABLA (thick full line), Bertini-Dresner (dotted line), CEM2k (thin dashed line), ISABEL-ABLA (thick dashed line).

in the very light evaporation residue region. In the region of the fission fragments, up to a factor of 3 is found between INCL4-ABLA and CEM2k models. A comparison between INCL4-ABLA and Bertini-Dresner shows that the quantity of elements produced are very similar for nuclides close to the target with differences in the isotopic distributions. For light elements, on the other hand, larger discrepancies are found between those two models. It was shown in the past that INCL4-ABLA predict well isotopic distribution of fission products for the reaction $\mathrm{p}+\mathrm{Pb}$ at $1 \mathrm{GeV}$ thus giving confidence in the results obtained with it for the chemical composition of the target in that particular region of mass.

The result obtained with a pure $\mathrm{Pb}-\mathrm{Bi}$ target has been computed with INCL4-ABLA spallation model. It is found (not shown) that the effect of impurities on the activity of the target is negligible.

In figure 4, we represent the amount of volatile gas produced in the lead bismuth target using models available in MCNPX2.5.0. Despite the fact that they are not major contributors to the activity of LBE, their interest relies on the fact that due to their radioactivity, they may be a source of concern in case of a containment failure. In particular, we have looked at the amount of hydrogen, helium, neon, argon, krypton, iodine and xenon produced in the target. It is found that the amount of hydrogen produced range from 2.7 litters with INCL4-ABLA to 4.1 litters with Bertini-Dresner. A factor 1.35 is found between those two models regarding helium production.

As for the main contributors to radioactivity due to volatile elements it is shown in figure 5 that ${ }^{83} \mathrm{Kr}$ metastable state (lifetime of 1.83 hours), ${ }^{127} \mathrm{Xe}$ (lifetime of 36.4 days) and tritium are the most important. We used for the calculation INCL4-ABLA model since it was already shown in the past [2] that fission product elements were correctly described by this model in that energy and mass range. In table 1 we show the quantity of polonium isotopes produced in LBE for several models. It is found that results are globally the same for ${ }^{210} \mathrm{Po}$ thus confirming the fact that this isotope is essentially 


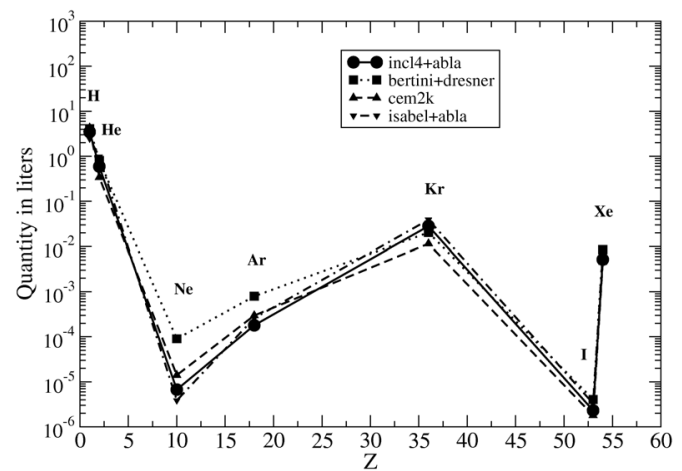

Fig. 4. Quantity of volatile gas produced in the $\mathrm{Pb}-\mathrm{Bi}$ target after 120 days of irradiation with $575 \mathrm{MeV}$ protons at $1.4 \mathrm{~mA}$ using several models.

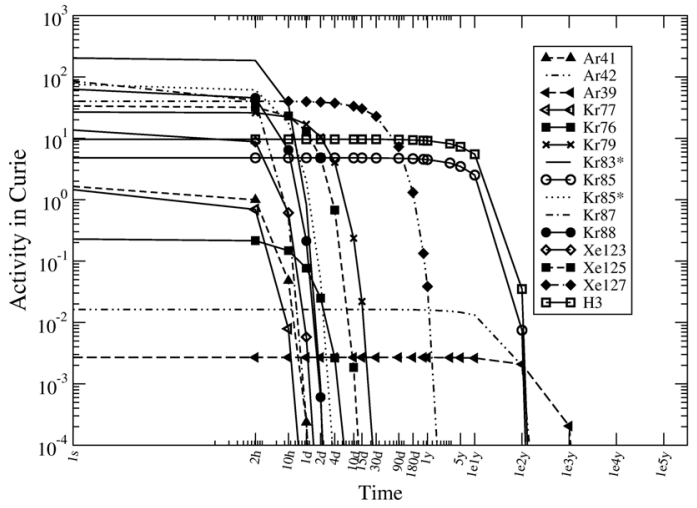

Fig. 5. Main contributors to the activity (in Curies) coming from volatile elements of the Megapie target as a function of cooling time after 120 days of irradiation with $575 \mathrm{MeV}$ protons at $1.4 \mathrm{~mA}$ using INCL4-ABLA.

Table 1. Quantity of polonium produced in grams for different spallation models.

\begin{tabular}{|c|c|c|c|c|}
\hline & $\begin{array}{c}\text { Incl4 } \\
- \\
\text { Abla }\end{array}$ & $\begin{array}{c}\text { Bertini } \\
-\end{array}$ & $\begin{array}{c}\text { CEM2 } \\
\mathrm{k}\end{array}$ & $\begin{array}{c}\text { Isabel } \\
- \\
\text { Abla }\end{array}$ \\
\hline${ }^{206} \mathrm{Po}$ & 0.011 & 0.015 & 0.011 & 0.012 \\
\hline${ }^{208} \mathrm{Po}$ & 0.079 & 0.097 & 0.116 & 0.079 \\
\hline${ }^{209} \mathrm{Po}$ & 0.052 & 0.015 & 0.083 & 0.036 \\
\hline${ }^{210} \mathrm{Po}$ & 1.036 & 1.067 & 1.084 & 1.142 \\
\hline
\end{tabular}

produced by activation by low energy neutrons. The same conclusion is drawn for the others isotopes except for ${ }^{209} \mathrm{Po}$.

\subsection{Container}

In the case of LBE container, just a few elements contribute to the total activity, mainly, ${ }^{51} \mathrm{Cr}$ (lifetime of 27.7 days) and ${ }^{55} \mathrm{Fe}$ (lifetime of 2.73 years) as shown in figure 6. They respectively come from activation by neutrons of ${ }^{50} \mathrm{Cr}$ and ${ }^{54} \mathrm{Fe}$. Activity saturates at around $1.4 \times 10^{4}$ curies when using INCL4-ABLA spallation models. In figure 7 are represented the modifications of the chemical composition of the container

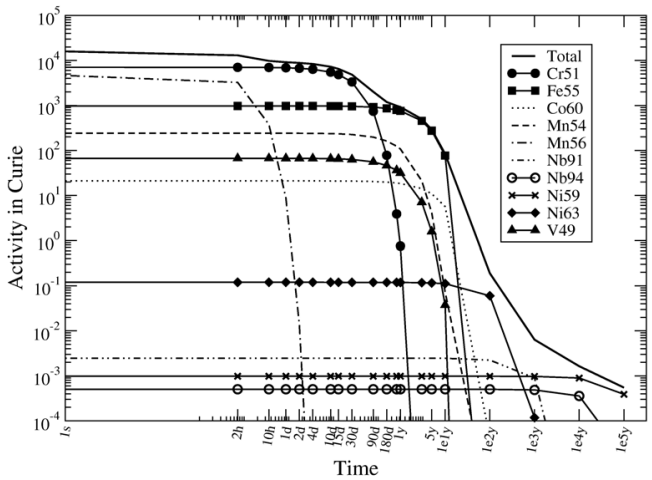

Fig. 6. Same as figure 1 for the container.

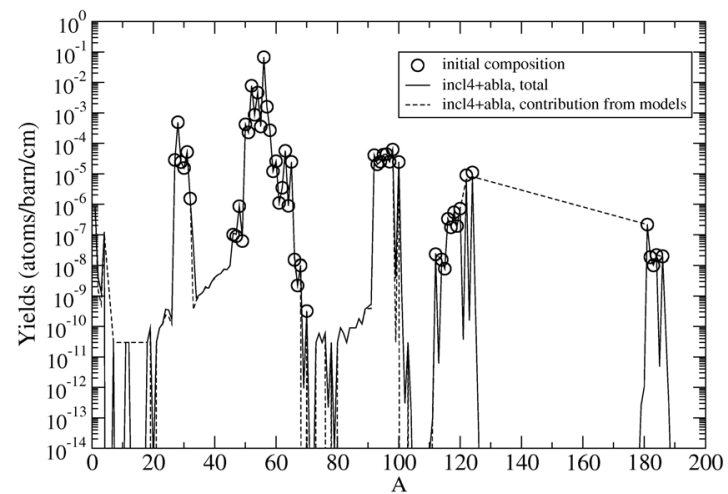

Fig. 7. Chemical composition of the container (window included) as a function of nuclide mass using INCL4-ABLA model and assuming 120 days of irradiation at $1.4 \mathrm{~mA}$ by $575 \mathrm{MeV}$ protons. Circles represent the initial composition and dotted line the contribution to the total yields coming from models.

(window included) after 120 days of irradiation at $1.4 \mathrm{~mA}$. The full line represents the chemical composition of the container after 120 days of irradiation whereas the dotted line shows the contribution coming from models only (i.e., reactions above $20 \mathrm{MeV}$ ). To obtain this last contribution, we have switched off the activation by low energy neutrons (below $20 \mathrm{MeV}$ ). It is found that most of elements produced in the container are coming from models thus confirming the fact that, in order to get the chemical composition of the target correctly, we have to develop spallation models as reliable as possible.

\subsection{Comparison LBE/Container}

A comparison between the activity of LBE and container is shown in figure 8 together with contributions coming from high-energy reactions only, using INCL4-ABLA models. It is found that for $\mathrm{Pb}-\mathrm{Bi}$ most of the activity comes from highenergy reactions. On the other hand, it is found that most of the activity of the container results from its activation by low energy neutrons. It is also worthwhile to notice that around 5 years of cooling, the activity of the container becomes of the same order of magnitude than that of lead-bismuth. 


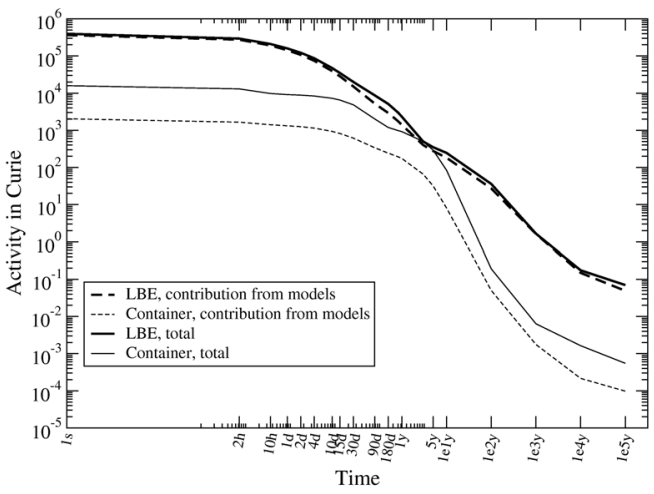

Fig. 8. Activity of the LBE and container (window included) due to high-energy reactions, calculated by models, (dashed lines) and total, adding the activation by low energy neutrons (full lines). The calculation is done using INCL4-ABLA in MCNPX.

\section{Conclusion}

The results presented in this paper indicate that the activity of liquid metal target comes mainly from spallation residues. As for the container, it is found that the activity is driven by activation by low energy neutrons (energy less than $20 \mathrm{MeV}$ ). In this case, the main contributors found are ${ }^{51} \mathrm{Cr},{ }^{55} \mathrm{Fe}$ and ${ }^{63} \mathrm{Ni}$ for period of times less than 27.7 days, between 27.7 days and 2.73 years, and above 2.73 years respectively. For the future, we are planning to extract damages produced, as atom displacements, in the window in order to give information on its lifetime.

This work was partially supported by the FP6 Euratom project EUROTRANS/NUDATRA, contract number FI6W-CT-2004-516520.

\section{References}

1. HINDAS (High- and Intermediate-energy Nuclear Data for Accelerator-driven Systems), FIKW-CT-2000-00031, European Commission contract.

2. A. Boudard et al., Phys. Rev. C 66, 044615 (2002).

3. A.R. Junghans et al., Nucl. Phys. A 629, 655 (1998).

4. R.E. Prael, LAHET Version 3.16, Report LA-UR-01-1655, June 18 (Los Alamos National Laboratory, Los Alamos, NM) (2001).

5. MCNPX User's Manual, Version 2.5.0, April 2005, LA-CP-050369, edited by Denise B. Pelowitz.

6. Y. Foucher, M. Vatré, L. Zanini (2006) (private communication).

7. H.W. Bertini, Phys. Rev. 131, 1801 (1963).

8. L. Dresner, Report ORNL-TM-196 (Oak Ridge National Laboratory, Oak Ridge, TN, 1962).

9. S.G. Mashnik, A.J. Sierk, Los Alamos National Laboratory report, LA-UR-01-5390 (2001).

10. Y. Yariv, Z. Praenkel, Phys. Rev. C 20, 2227 (1979).

11. L. Donadille et al., Contribution to Int. Conf. AccApp'03, San Diego, USA, June 2003 (2003). 